\title{
Selective Solar Concentrators for Biofuel Production and Photovoltaic Applications
}

\author{
Nima Talebzadeh, Paul G. O'Brien \\ Department of Mechanical Engineering, Lassonde School of Engineering, York University \\ 4700 Keele Street, Toronto, Ontario, M3J 1P3, Canada \\ nimatg@yorku.ca; paul.obrien@lassonde.yorku.ca
}

\section{Extended Abstract}

Algae-based biofuels have become of increasing interest in recent years as a renewable energy source to replace energy derived from fossil fuels. Algae exhibits remarkable potential for producing large amounts of energy, for example as much as $60 \%$ of their Biomass can be converted to oil, with 30 to 50\% more energy output per gallon than gasoline [1]. Algae also generates about $60 \%$ of the Earth's atmospheric oxygen and, in good cultivation conditions, algae produces protein and energy biomass 30 to 100 times faster than land plants [1-3]. Furthermore, algae does not require the entire incident solar spectrum to perform photosynthesis. That is, algae primarily utilizes the blue and red portions of the solar spectrum, referred to as Photosynthetic Active Radiation (PAR), while a large portion of the green and near-infrared light received from the sun is not used in the photosynthetic reaction [4]. In this context, incident solar radiation can be utilized for agrivoltaic applications [5]

This study discusses a novel, low cost and efficient solar spectrum splitter that partitions solar radiation into its PAR and non-PAR components. The incident PAR and non-PAR solar irradiance is used to simultaneously drive biofuel production and photovoltaic cells, respectively. For this purpose, photonic micro/nano structures are integrated into solar spectrum splitters that transmit PAR to enable underlying algae cultivation, while concentrating non-PAR at the side-walls of the solar spectrum splitter to power photovoltaic cells. Moreover, in this study we also investigate the benefits of utilizing the aforementioned solar spectrum splitter in energy efficient agrivoltaic greenhouses that generate photovoltaic power while producing crops.

In this work wave-optics analysis is used to model a solar spectrum splitter made from transparent panels that utilize photonic micro/nanostructures on its surface in conjunction with the total internal reflectance (TIR) phenomenon to separate and direct non-PAR to the side walls of the panel. The wave-optics analysis investigates different photonic micro/nano structures, such as metasurfaces and diffraction gratings integrated into the surface of the solar spectrum splitter. Simulation results reveal that this wavelength selective solar concentrator technology is a novel approach wherein algae can be grown under protected, controlled conditions, while the cost of operations is offset by the structure's internal electrical production, without any significant loss to the algal growth rate or achievable biomass density.

\section{References}

[1] M. R. Edwards, Green Algae Strategy End Oil Imports and Engineer Sustainable Food and Fuel. Tempe, Arizona, CreateSpace, 2008.

[2] D. R. Georgianna1 and S. P. Mayfield, "Exploiting diversity and synthetic biology for the production of algal biofuels," Nature, vol. 488, no. 7411, pp. 329-335.

[3] D. Surendhiran and M. Vijay "Microalgal Biodiesel - A Comprehensive Review on the Potential and Alternative Biofuel," Res. J. Chem. Sci., vol. 83, no. 11, pp. 71-82, 2012.

[4] T. B. Arp, Y. Barlas, V. Aji, and N. M. Gabor, "Natural Regulation of Energy Flow in a Green Quantum Photocell," Nano Lett., vol. 16, pp. 7461-7466, 2016.

[5] C. J. M. Emmott, J. A. Rohr, M Campoy-Quiles, T. Kirchartz, A, Urbina, N. J. Ekins-Daukes, J. Nelson, "Organic Photovoltaic Greenhouses: A Unique Application for Semi-Transparent PV,” Energy Environ. Sci., vol. 8, pp. 1317$1328,2015$. 